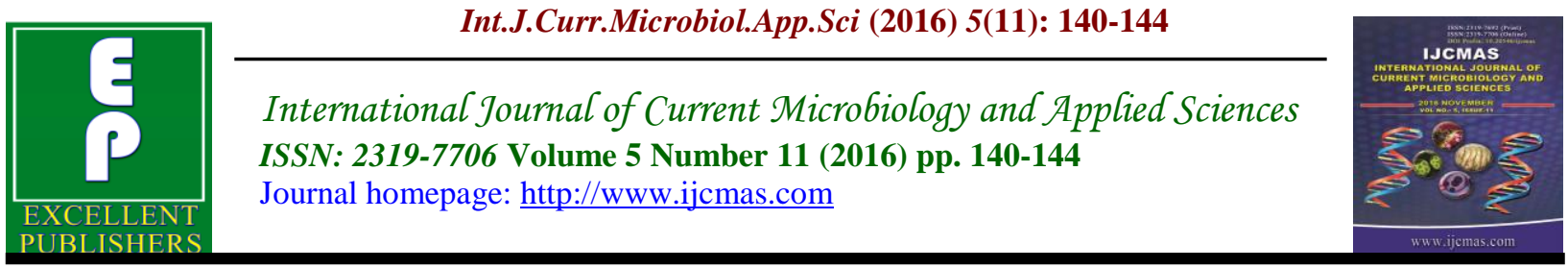

Short Communication

http://dx.doi.org/10.20546/ijcmas.2016.511.016

\title{
Resistance in Neisseria gonorrhoeae: A Cause of Concern?
}

\author{
Kirti Nirmal $^{1}$, Rumpa Saha ${ }^{1}$, V.G. Ramachandran ${ }^{1}$, Shukla Das ${ }^{1}$ and S.N. Bhattacharya ${ }^{2}$ \\ ${ }^{1}$ Department of Microbiology, University College of Medical Science \& Guru Tag Bahadur \\ Hospital, Delhi-110095, India \\ ${ }^{2}$ Department of Dermatology Venereology and Leprology, University College of Medical \\ Science \& Guru Tag Bahadur Hospital, Delhi-110095, India \\ *Corresponding author
}

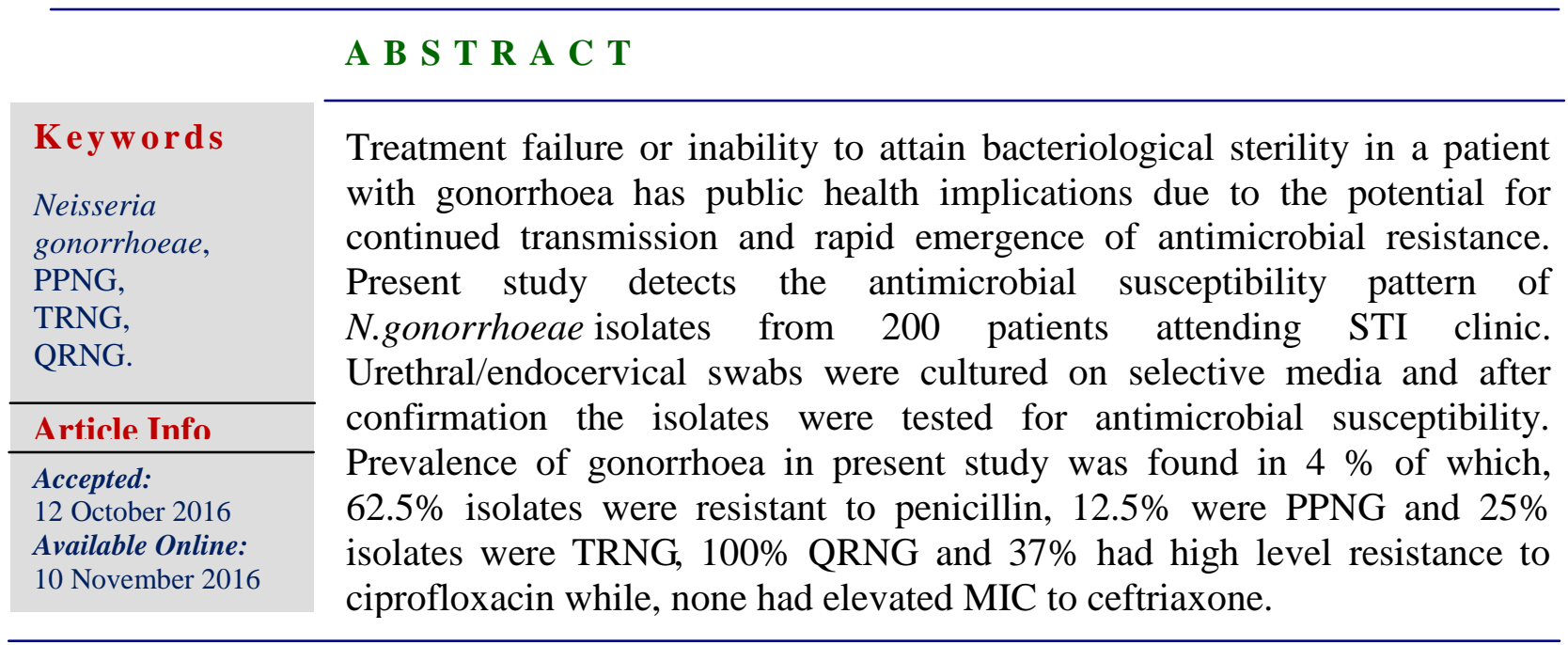

\section{Introduction}

Gonorrhoea, a disease well documented from ancient times, continues to defy man's attempt to control it. It is a successful pathogen with a remarkable journey over the years. It has been adept at developing resistance to several commonly used antimicrobials (Bala et al., 2005).

The failure to cure a case of gonorrhoea has public health implications due to the potential for continued transmission and rapid emergence of antimicrobial resistance. Moreover, it also facilitates the spread of HIV. The present study was initiated to document the resistance pattern of N.gonorrhoeae isolates from patients attending STI clinic at a large tertiary care hospital in East Delhi.

\section{Experimental procedure}

Two hundred consecutive patients, who attended the STI clinic of our hospital, were enrolled in the study. Urethral and endocervical swabs were collected from male and female patients respectively, with a suggestive history. Samples were subjected to direct examination by gram staining and culture isolation on chocolate agar (CA) with vancomycin, colistin, nystatin and trimethoprim supplements and incubated with 5-10\% carbon dioxide for 
24-48 hours at $37^{\circ} \mathrm{C}$. A presumptive diagnosis of gonococcal urethritis was made on observing polymorphonuclear leucocytes with gram-negative intracellular diplococci. After confirming the isolates as N.gonorrhoeae by rapid carbohydrate utilization test, antimicrobial susceptibility testing (AST) of N.gonorrhoeae was done on CA by disc diffusion technique following the Antimicrobial Gonococcal Survillance Programme (AGSP) method (based on CDS technique) using penicillin $(0.5 \mathrm{IU})$, tetracycline $(10 \mu \mathrm{g})$, ceftriaxone $(0.5 \mu \mathrm{g})$, spectinomycin $(100 \mu \mathrm{g})$, ciprofloxacin $(1 \mu \mathrm{g})$, nalidixic acid $(30 \mu \mathrm{g})$, azithromycin $(15 \mu \mathrm{g})$ and cefpodoxime $(10 \mu \mathrm{g})$ (Oxoid, U.K). The annular radius of zone of inhibition was compared with interpretive standards and used to label isolates as sensitive / less sensitive / resistant. All the isolates were also tested for beta lactamase production by chromogenic cephalosporin disc method (Oxoid, U.K).

MIC of ceftriaxone was determined by $\mathrm{E}$ strips containing $0.002-32 \mu \mathrm{g} / \mathrm{ml} \quad(\mathrm{AB}$ Biodisks, Sweden) \& based on MIC value isolates were labelled as ceftriaxone sensitive $(\mathrm{MIC} \leq 0.03 \mu \mathrm{g} / \mathrm{ml}) \&$ less sensitive ( $\mathrm{MIC} \geq 0.06 \mu \mathrm{g} / \mathrm{ml}$ ).

MIC of ciprofloxacin was determined by Estrips containing 0.002-32 $\mu \mathrm{g} / \mathrm{ml}$ (Difco, USA) \& based on MIC value isolates were labelled as ciprofloxacin sensitive (MIC $\leq$ $0.03 \mu \mathrm{g} / \mathrm{ml}$ ) and less sensitive (MIC between $0.06-0.5 \mu \mathrm{g} / \mathrm{ml}$ ) \& resistant (MIC $\geq 1.0$ ). WHO strains A to E obtained from WHO GASP SEAR Regional Reference Lab, New Delhi were used for quality control for disc diffusion and MIC testing.

\section{Results and Discussion}

In present study, all patients which were suggestive of gonorrhoea by gram stain isolated N.gonorrhoeae on culture.
Overall gonococcal urethritis was detected in $4 \%$ of our patients with a female predominance $(\mathrm{M}: \mathrm{F}=3: 5)$. These female patients were in the sexually active age group of 20-35 years. Most of the patients with gonorrhoea (75\%) presented mainly with discharge which was thick and yellow in females \& mucopurulent in male patients.

Penicillin resistance was observed in $62.5 \%$ of isolates of which $12.5 \%$ were Penicillin producing $N$. gonorrhoeae (PPNG). All PPNG isolates were found to be ciprofloxacin resistant. $25 \%$ of isolates were TRNG, but sensitive to other antibiotics. None of the isolates had elevated MIC to ceftriaxone. All isolates were resistant to ciprofloxacin and $37 \%$ among them had high level resistance to ciprofloxacin (MIC $\geq 8 \mu \mathrm{g} / \mathrm{ml}$ ). (Fig 1, Fig 2)

The number of patients with gonorrhoea at $4 \%$ in this study is much less when compared to an earlier study from the same location with a figure of $19.3 \%$ in the year 2010 (Choudhry et al., 2010). Prevalence of gonorrhoea among STIs clinic attendees in different regions of India varies from 3-19 $\%$ (Sharma et al., 2004). A higher rate of gonorrhoea in women is comparable to study from Mumbai (9.7\%) and probably relates to their ignorance \& stigma attached to the disease (Divekar et al., 2000).

Our observation also underscores the emergence of penicillin resistance in N.gonorrhoeae isolates. Our data of PPNG at $12.5 \%$ is comparable to $8-66 \%$ reported from other parts of India and SEAR countries (Sunil et al., 2006; Rahman et al., 2002)). PPNG at $88 \%$ in North India was documented during 2011-2013 (Bharara et al., 2015) and in WHO western pacific region their prevalence varies from 1\% -90 $\%$ (Tapsall et al., 2002). This brisk extension of PPNG is due to the dissemination of 4.4 
$\mathrm{MDa}$ and 24.5 $\mathrm{MDa}$ plasmids associated with the Asian strains of N.gonorrhoeae.

Quinolone resistant $N$.gonorrhoeae (QRNG) is on the rise as evidenced by the present study $(100 \%)$ and other reports from northern India (Sunil et al., 2006; Bala et al.,2007; Bharara et al.,2015), thus, periodic monitoring is necessary. High QRNG is also documented from Bangladesh, Pakistan and
Bhutan (Ray et al., 2005; Jabeen et al., 2011). Elevated prevalence of QRNG probably indicates unregulated and misuse of these over-the-counter drug, and hence quinolone are no longer recommended for the treatment for gonorrhoea in India. Inappropriate use of this drug by malapropos health-care providers in South East Asia is a cause of great concern.

Fig.1 AST of N.gonorrhoeae.

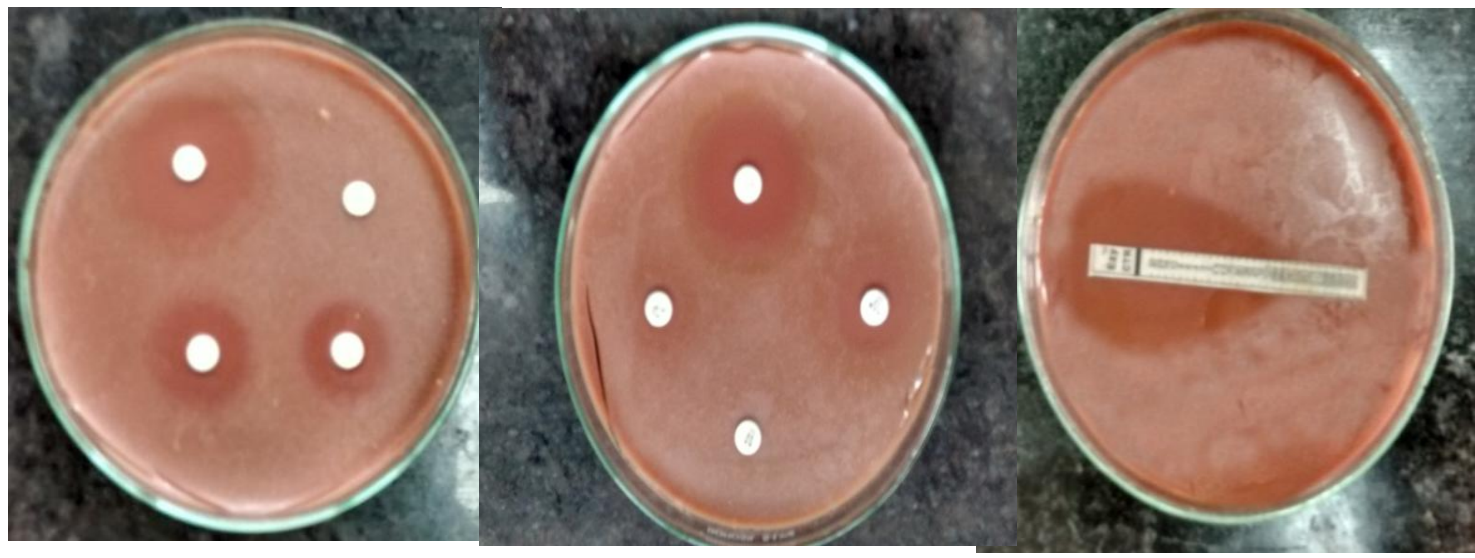

A1

A2

A3

Fig.2 Antibiotic resistance pattern of N.gonorrhoeae strain.

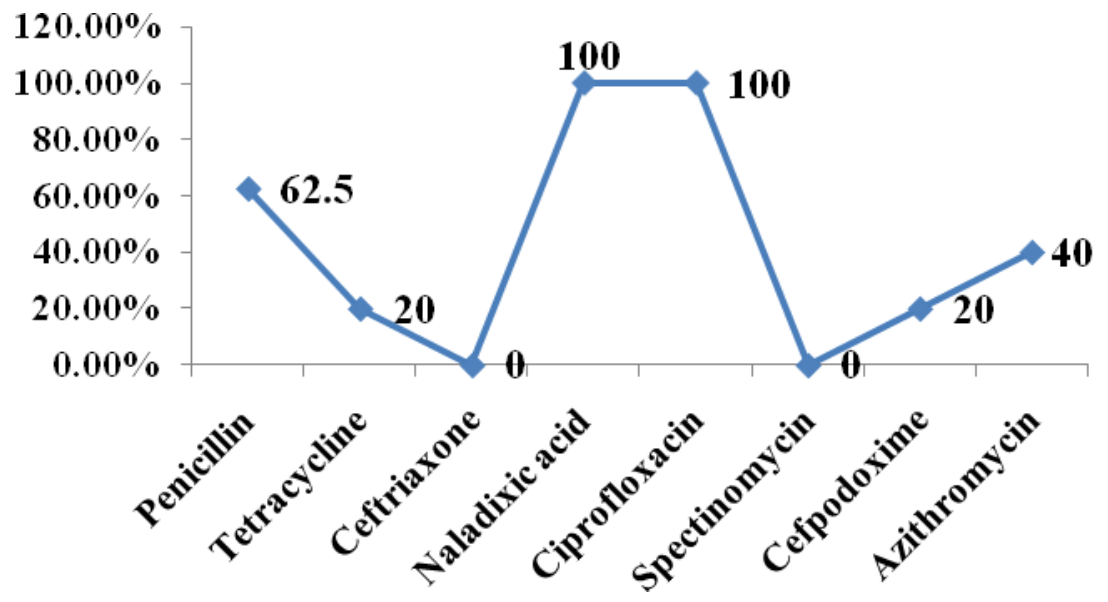

TRNG has been documented to vary from 12 to $51 \%$ in North India (Bharara et al., 2015; Sunil et al., 2013). Our result (25\%) is comparable with the above data. Reports from South India document 33.8-45.6\% TRNG (Ray et al., 2005). Bangladesh and Pakistan have recorded higher TRNG (Ray et al., 2005; Jabeen et al., 2011).Location of 
tet $\mathrm{M}$ gene on the transferable plasmid along with ongoing selective pressure due to the use of tetracycline in syndromic management of STIs as also to treat other clinical conditions may account for efficient transmission of resistance reflecting high TRNG.

Although decreased susceptibility to ceftriaxone (MIC $\geq 0.06$ to up to $0.25 \mu \mathrm{g} / \mathrm{ml}$ ) has been reported from Delhi (Bala et al., 2007; Bharara et al., 2015), as early as 2001, Japan has reported resistance to extended spectrum cephalosporin even earlier (Muratani et al., 2001). Similar reports followed from other countries (Tarsall et al., 2006). Present study did not observe increased MIC to ceftriaxone. This however is still of concern since evolution of resistance has been shown to precede treatment failure complicating effective disease control in particular geographical regions.

In conclusion, clinical suspicion of gonorrhoea must be supplemented with laboratory isolation of the etiological agent for AST to guide appropriate treatment. It is recommended that the treatment of gonorrhoea should be with drugs which have efficacies that approach $100 \%$ \& treatment with efficacies $<95 \%$ should never be used (Aggarwal et al., 2002). In the light of high QRNG, TRNG \& emergence of decreased susceptibility to extended spectrum cephalosporin, prudent use of drugs coupled with periodic monitoring of AST of consecutive GC isolates would help to preserve antibiotic effectiveness in the treatment of uncomplicated gonorrhoea and also prevent dissemination of drug resistant strains in the community. Continuous surveillance of antimicrobial resistance is thus crucial to tailor treatment schedules when the need arise and incorporate this activity as a necessary operating procedure.

\section{References}

Aggrawal, S., Garg, V.K., Aggarwal, A., Joshi, A., Ramachandran, V.G. 2002. Nepal J. Dermatol. Venerol. Leprol., 2: $17-19$.

Bala, M., Ray, K., Gupta, S.M. 2005. Comparison of disc diffusion results with minimum inhibitory concentration (MIC) values for antimicrobial susceptibility testing of Neisseria gonorrhoeae. Indian J. Med. Res., 122: 48-51.

Bala, M., Ray, K., Gupta, S.M., Muralidhar, S. and Jain, R.K. 2007. Changing trends of antimicrobial susceptibility patterns of Neisseria gonorrhoeae in India and the emergence of ceftriaxone less susceptible N. gonorrhoeae strains. J. Antimicrobial Chemother., 60: 582-586.

Bharara, T., Bhalla, P., Rawat, D., Garg, V.K., Sardana, K., Chakravarti, A. 2015. Rising trend of antimicrobial resistance among Neisseria gonorrhoeae isolates and the emergence of $N$. gonorrhoeae isolate with decreased susceptibility to ceftriaxone. Indian J. Med. Microbiol., 33: 39-42.

Choudhry, S., Ramachandran, V.G., Das, S., Bhattacharya, S.N., Mogha, N.S. 2010. Pattern of sexually transmitted and predominance of syndromic management against etiological diagnosis in patients attending the sexually transmitted infection clinic of a tertiary care hospital. Indian J. Sex Transm. Dis., 31: 104-8.

Divekar, A.A., Gogate, A.S., Shivkar, L.K., Gogate, S., Badhwar, V.R. 2000. Disease prevalence in women attending the STD clinic in Mumbai (formerly Bombay), India. Int. J. STD AIDS, 11: 45-8. 
Jabeen, K., Nizamuddin, S., Irfan, S., Erum, Khan, Faisal Malik, Afia Zafar. 2009. Increasing trend of resistance to penicillin, tetracycline, and fluoroquinolone resistance in Neisseria gonorrhoeae from Pakistan (19922009). J. Trop. Med., 960501.

Muratani, T., Akasaka, S., Kobayashi, T., Yamada, Y., Inatomi, H., Takahashi K et al. 2001. Outbreak of cefozopran (penicillin, oral cephems and aztreonam)-resistant Neisseria gonorrhoeae in Japan. Antimicrob Agents Chemother., 45: 3603-6.

Rahman, M., Sultan, Z., Monira, S., Nagpal, P., Sachdeva, D., Bala, M. et al. 2002. Antimicrobial susceptibility of Neisseria gonorrhoeae isolated in Bangladesh (1997 to 1999): rapid shift to fluoroquinolone resistance. J. Clin. Microbiol., 40: 2037-40.

Ray, K., Bala, M., Kumari, S., Gupta, S.M., Gupta, P. 2005. Antimicrobial resistance of Neisseria gonorrhoeae in selected World Health Organization Southeast Asia Region countries: an overview. Sex Transm. Dis., 32: 178184. 35 .
Sharma, V.K., Khandpur, S., Gupta, N., Goyal, A. 2004. Changing pattern of sexually transmitted infection in India. Natl. Med. J. India, 17: 310-19.

Sunil, S., Dharmendra, S., S.D. Mehta, Bhupinder, S., Manu, S., Bhushan K et al. 2006. Emergence of ciprofloxacin resistant Neisseria gonorrhoeae in north India, Indian J. Med. Res., 123, pp 707-710.

Sethi, S., Golparian, D., Bala, M., Dorji, D., Ibrahim, M., Jabeen, K., et al. 2013. Antimicrobial susceptibility and genetic characteristics of Neisseria gonorrhoeae isolates from India, Pakistan and Bhutan in 2007-2011. BMC Infect. Dis., 13: 35.

Tapsall, J.W. 2003. Surveillance of antibiotic resistance in Neisseria gonorrhoeae in the WHO Western Pacific Region 2002. Commun. Dis. Intell., 27: 488-91.

Tapsall, J.W. 2006. Annual report of the Australian Gonococcal Surveillance Programme, 2005. Commun. Dis. Intell., 30: 205-10.

\section{How to cite this article:}

Kirti Nirmal, Rumpa Saha, V.G. Ramachandran, Shukla Das and S.N. Bhattacharya. 2016. Resistance in Neisseria gonorrhoeae: A Cause of Concern? Int.J.Curr.Microbiol.App.Sci. 5(11): 140-144. doi: http://dx.doi.org/10.20546/ijcmas.2016.511.016 